\title{
Diacronie
}

Studi di Storia Contemporanea

$\mathrm{N}^{\circ} 32,4 \mid 2017$

Proiezioni individuali e agire collettivo nella storia

\section{Nota introduttiva n. 32 - dicembre 2017}

Jacopo Bassi, Luca Bufarale e Luca Zuccolo

\section{(2) OpenEdition}

\section{Journals}

\section{Edizione digitale}

URL: http://journals.openedition.org/diacronie/6458

DOI: $10.4000 /$ diacronie. 6458

ISSN: 2038-0925

\section{Editore}

Association culturelle Diacronie

Notizia bibliografica digitale

Jacopo Bassi, Luca Bufarale e Luca Zuccolo, « Nota introduttiva n. 32 - dicembre 2017 », Diacronie [Online], N 32, 4 | 2017, Messo online il 29 décembre 2017, consultato il 24 septembre 2020. URL : http://journals.openedition.org/diacronie/6458; DOI : https://doi.org/10.4000/diacronie.6458

Questo documento è stato generato automaticamente il 24 settembre 2020.

Creative Commons License 


\section{Nota introduttiva n. 32 - dicembre 2017}

Jacopo Bassi, Luca Bufarale e Luca Zuccolo

Proiezioni individuali e agire collettivo nella storia Ruoli sociali, aspetti politici e nodi storiografici tra pubblico e privato 
1 Il numero si apre con il saggio di Benedetta Giuliani dedicato ad un'analisi di alcune nuove tendenze nella storiografia di area anglosassone. Giuliani si sofferma in particolare sui percorsi di ricerca più innovativi e dibattuti: la public history e la applied history. Il saggio prende in esame con particolare attenzione le attività del network di ricerca History \& Policy e dell'Applied History Project del

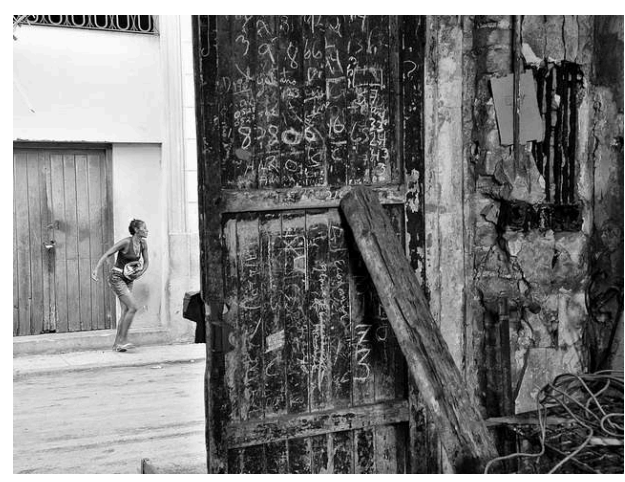
Belfer Center. Ciò che emerge ripercorrendo il dibattito è la necessità di riflettere sul ruolo della storia e sulla sua attuale funzione.

2 Calogero Laneri affronta il complesso rapporto tra il Partito comunista italiano e il processo di unificazione europea nella fase 1979-84, dalla fine dei governi di solidarietà nazionale alla scomparsa del carismatico leader Enrico Berlinguer, avvenuta alcuni giorni prima delle elezioni europee in cui il partito arrivò per la prima a sorpassare in termini di voti la Democrazia cristiana. Prendendo in considerazione soprattutto la stampa di partito, l'autore sottolinea il superamento delle pregiudiziali antieuropeiste del passato, analizzando le posizioni dei dirigenti più noti e di alcuni intellettuali e soffermandosi anche sul riavvicinamento con il leader europeista Altiero Spinelli. Viene rimarcata la peculiarità dell'europeismo del PCI, fondato sulla riduzione degli squilibri economici tra gli stati membri e su una posizione di equilibrio tra i due blocchi. Emergono, però, anche le contraddizioni e le fragilità di questo progetto, che di lì a pochi anni diventeranno palesi con il superamento della guerra fredda e la fine stessa del PCI.

3 L'articolo di Martina Crescenti ci propone un interessante punto di vista sulla società turca analizzando il ruolo della donna all'interno del panorama politico islamico. Il saggio mette infatti in luce l'importanza della donna nel programma politico dei movimenti e dei partiti islamici in Turchia in quanto figura di trasmissione dei valori islamici sia nella sfera privata e familiare sia nella sfera pubblica, palesandone il ruolo chiave nell'ascesa e nel consolidamento dell'attuale realtà politica islamica.

4 Carlo Bianchi prende invece in esame l'Italia della seconda metà degli anni Settanta: un paese scosso dal terrorismo, già incamminato sulla via del riflusso, che vede imporsi nel panorama della popular music Angelo Branduardi. Il cantautore, con testi di stampo favolistico avvolti da un misto di sonorità etniche e arcaiche, si inserisce nell'ultima fase del progressive. Le teorie di Guy Debord e Pierre Bourdieu - sostiene Bianchi soprattutto prendendo in considerazione il rapporto "circolare" che Bourdieu individua fra artista e società, offrono elementi per una coerente spiegazione del fenomeno Branduardi, abilmente pilotato dal manager David Zard. Le teorie del sociologo francese aiutano a comprendere questo meccanismo contestualizzandolo in una temperie culturale e interpretando le scelte di un artista continuamente in bilico fra personale e collettivo.

5 Il saggio di Giovanna Savant è focalizzato sulla visione che il Partito socialista italiano aveva degli eventi rivoluzionari della Russia del 1917. Così come nel saggio di Laneri, anche in questo caso la stampa di partito è la fonte principale. L'autrice ricostruisce il dibattito che si aprì tra i vari dirigenti sulle tappe della rivoluzione, dal rovesciamento 
dello zarismo sino al trattato di Brest-Litovsk. La sua analisi evidenzia come le divergenze di valutazione e talvolta le oscillazioni e i cambiamenti di opinione in materia - certo attribuibili in parte alle difficoltà di reperire informazioni affidabili su quanto stava avvenendo in quel paese - costituiscano anche un'occasione ineludibile per l'elaborazione teorica sulla via al socialismo, oltre che una sorta di cartina tornasole delle fratture tra le varie correnti del PSI.

6 La seconda sezione (II. Laboratorio) propone una nuova edizione del Laboratorio, uno spazio dedicato agli elaborati degli studenti dei corsi di laurea magistrale. Il tema dei cinque saggi - selezionati e curati da Eloisa Betti e Maria Pia Casalena - presentati in questo numero è il "Lavoro delle donne", come viene esplicitato nell'introduzione alla sezione.

7 Chiude questo numero il consueto spazio dedicato alle recensioni (III).

8 Il coordinamento redazionale è stato svolto da Jacopo Bassi, Luca Bufarale, Luca Zuccolo.

9 Un ringraziamento particolare a Leonardo Recupero e Claudio Sessa per il contributo alla curatela del numero.

10 Buona lettura,

11 Jacopo Bassi, Luca Bufarale, Luca Zuccolo

\section{AUTORI}

\section{JACOPO BASSI}

Jacopo Bassi ha conseguito la Laurea Triennale in «Storia del mondo contemporaneo» presso l'Università di Bologna sostenendo una tesi in Storia e istituzioni della Chiesa ortodossa dal titolo Tra Costantinopoli e Atene: Il passaggio delle diocesi dell'Epiro all'amministrazione della Chiesa di Grecia e la 'Praxis' del 1928; presso lo stesso ateneo, nel 2008, ha discusso la tesi specialistica in Storia della Chiesa dal titolo Epiro crocifisso o liberato? La Chiesa ortodossa in Epiro e in Albania meridionale nel XX secolo (1912-1967). Attualmente collabora con le case editrici Il Mulino e Zanichelli.

URL: < http://www.studistorici.com/2009/02/24/jacopo_bassi/ >

\section{LUCA BUFARALE}

Luca Bufarale ha conseguito nel 2008 la Laurea Specialistica in Storia d'Europa (indirizzo contemporaneo) presso l'Università di Bologna e nel 2012 il Dottorato di Ricerca in Scienze storiche presso l'Università di Padova. Dopo aver condotto i suoi primi studi sulle trasformazioni economiche e i progetti di riforma nell'Unione Sovietica degli anni Sessanta, si è dedicato prevalentemente alla ricostruzione della figura del dirigente socialista italiano Riccardo Lombardi. I suoi interessi spaziano dalla storia dei movimenti politici alla storia delle politiche economiche. È autore del volume Riccardo Lombardi. La giovinezza politica 1919-1949 (Roma, Viella, 2014). Attualmente è docente supplente di storia e filosofia nei licei e segue un progetto di ricerca per l'Associazione Labour "Riccardo Lombardi".

URL: < http://www.studistorici.com/2010/12/02/luca-bufarale/ > 


\section{LUCA ZUCCOLO}

Luca Zuccolo, dopo aver conseguito la laurea in Storia Contemporanea e il titolo di dottore magistrale in Storia d'Europa presso l'Università di Bologna (2005 e 2008), ha ottenuto il titolo di Dottore di ricerca in Storia Contemporanea presso il SUM - Istituto Italiano di Scienze Umane Napoli. I suoi campi di ricerca sono: la modernizzazione dell'Impero Ottomano (XIX-XX secolo), il ruolo della stampa ottomana nel contesto imperiale ed europeo e i movimenti sociali che hanno preparato l'avvento della società turca contemporanea.

URL: < http://www.studistorici.com/2010/12/01/luca-zuccolo/ > 